\title{
THE PREVENTION OF NEURAL TUBE DEFECTS BY FOLIC ACID SUPPLEMENTATION
}

\section{The Director-General, Department of National Heaith \& Population Development has requested us, in view of its general public health significance, to publish the following statement from his department.}

Neural tube defects, in particular spina bifida and anencephaly, are serious and relatively common congenital abnormalities worldwide. They also occur in South Africa and affect all population groups to varying degrees. The overall incidence in South Africa is approximately $1-2$ per 1000 newborns. Higher incidences, up to 6 per 1000 newborns have been recorded in certain parts, especially in some rural areas of the country. In total as many as $\mathbf{1 5 0 0}$ newborns could be affected by a neural tube defect each year. The precise aetiology of neural tube defects is still unknown.

Most anencephalic babies are stillborn and the remainder usually die within the first day. The mortality rate among patients with spina bifida is high. Many such patients can be treated, which includes repeated surgery and hospitalization, as well as antibiotic and orthopaedic management. Although treatment may be successful, most children with spina bifida will remain handicapped to a greater or lesser degree, both physically and/or mentally. They will continue to require life-long supportive medical services and care, as well as special schooling.

The Department of National Health and Population Development ranks the prevention of neural tube defects as a high health service priority and fully supports measures designed for the prevention of neural tube defects. One such method of prevention is by way of maternal serum screening tests and prenatal diagnosis. Whereas this procedure remains an important option for women who have access to such screening and diagnostic services, a new supplementary method of primary prevention has recently become available. Experimental and epidemiological evidence has shown that periconceptual dietary supplementation with folic acid can substantially decrease the incidence of neural tube defects. Based on these scientific results, health authorities and professional bodies in various countries have oficially expressed themselves in favour of periconceptual dietary folic acid supplementation to substantially reduce the risk of babies being affected with neural tube defects.

The Department of National Health and Population Development has taken note of this important development and especially of the scientific evidence in support of the recommended procedure (see publications listed below). In view of the proven beneficial effect of folic acid supplementation on the reduction of the incidence of neural tube defects, this Department strongly recommends that all women of childbearing age be informed of the benefits of periconceptual folic acid supplementation and be encouraged to make use of this treatment. In particular, it is recommended that women who already had a baby or close family member affected with a neural tube defect, be advised to use folic acid supplementation periconceptually when planning a subsequent pregnancy. According to published recommendations all women of childbearing age who are capable of becoming pregnant should consume 0,4 $\mathrm{mg}$ of folic acid per day for the purpose of reducing their risk of having a pregnancy affected with a neural tube defect. It is trusted that the medical profession, other health service providers and authorities countrywide will support the endeavour to reduce the risk for neural tube defects by the way of the procedure as recommended.

\section{REFERENCES}

MILUNSKY A, et al (1989). Multivitamin/Folic acid supplementation in early pregnancy reduces the prevalence of neural tube defects. $J$ Am Med Ass, 262: 2847 - 2852.

MRC VITAMIN STUDY RESEARCG GROUP (Wald N) (1991). Prevention of neural tube defects: Results of the Medical Research Council Vitamin Study. Lancet, 338 (No 8760 ): 131-137.

EDITORIAL (1991). Folic acid and neural tube defects. Lancet, 338 (No 8 760): 153-154.

CDC (1992). Recommendations for the use of folic acid to reduce the number of cases of spina bifida and other neural tube defects. Morbidity and Mortality Weekly Report, U.S. Department of Health and Human Services, Public Health Service, CDC, 41, No. RR - 14: 1 - 7

LEADS FROM THE MMWR (1992; 41: RR-14). Recommendations for use of folic acid to reduce number of spina bifida cases and other neural tube defects. JAm Med Ass, 269: 1233 - 1 238.

WERLER M W, et al (1993). Periconceptional folic acid exposure and risk of occurrent neural tube defects. J Am Med Ass, 269: 1257 - 1 261.

EDITORIAL (1993). Folic acid preventable spina bifida and anencephaly. J Am Med Ass, 269: 1 292 - 1293. 